\title{
VACUUM KERR-SCHILD METRICS GENERATED BY NONTWISTING CONGRUENCES ${ }^{\dagger}$
}

László Á. Gergely ${ }^{a}$ ) and Zoltán Perjés $\left.{ }^{b}\right)$

\begin{abstract}
The Kerr-Schild pencil of metrics $\tilde{g}_{a b}=g_{a b}+V l_{a} l_{b}$, with $g_{a b}$ and $\tilde{g}_{a b}$ satisfying the vacuum Einstein equations, is investigated in the case when the null vector $l$ has vanishing twist. This class of Kerr-Schild metrics contains two solutions: the Kasner metric and a metric wich can be obtained from the Kasner metric by a complex coordinate transformation. Both are limiting cases of the Kóta-Perjés metrics. The base space-time is a pp-wave.
\end{abstract}

Keywords: General Relativity, Kerr-Schild metrics, nontwisting null congruences

\section{INTRODUCTION}

The Kerr-Schild map

$$
\tilde{g}_{a b}=g_{a b}+V l_{a} l_{b}
$$

generates a pencil of space-time metrics $\tilde{g}_{a b}$ from the metric $g_{a b}$ where $l$ is a null vector and $V$ a function. The solution of the original problem, where a flat parent space-time is mapped to a vacuum space-time, has been known for some time. In the case of KerrSchild pencils with a non-twisting and divergence-free $l$, this is due to Trautman ${ }^{1}$. For the congruences with nonvanishing divergence or twist, the general solution is given by Kerr and Schild ${ }^{2}$.

Here we shall not impose any restriction on the parent metric $g$ apart from being Lorentzian and vacuum. Still, it follows from the vacuum Einstein equations for the pencil (0) that the null vector $l$ is tangent to geodesics. The general solution of this problem was given recently by us $^{3,4,5}$. However, the special case when $l$ is twist-free is not per se covered by the generic procedure, and hints only were given in Ref. 5 as to how to treat the twist-free fields. The full details are given in this paper.

$\dagger$ Research supported by OTKA fund no. 1826 and by the Pro Renovanda Cultura Hungariae Foundation

$\left.{ }^{a}\right)$ Department of Theoretical Physics, Jzsef Attila University, H-6720 Szeged, Aradi vrtank tere 1, Hungary

$\left.{ }^{b}\right)$ Central Research Institute for Physics, ${ }_{1}$-1525 Budapest 114, P.O.Box 49, Hungary 
In section 2 we state a theorem characterizing the twist-free Kerr-Schild metrics by the value of the shear parameter $\eta$. As in the generic case, the values $\sin \eta=0, \pm 1, \pm \frac{1}{\sqrt{2}}$ of the shear parameter are exceptional, and these metrics cannot be continuously extended to other values of $\eta$. These exceptional cases are covered by the treatment in Ref. 5, letting the twist vanish. However, the generic treatment in Ref. 5 cannot be extended to the fields with arbitrary $\eta$. Sections 3 and 4 are devoted to these fields. Nonetheless, the resulting metrics prove to be the twist-free limits of the generic metrics ${ }^{5}$.

\section{SOLUTION OF THE $\Psi_{1}$ SYSTEM}

The computations follow closely the pattern of the generic case where the NewmanPenrose formalism ${ }^{6}$ was used. The tetrad vectors are denoted $l=D, n=\Delta, m=\delta$, and $\bar{m}=\bar{\delta}$. The expressions for the optical scalars, the curvature quantity $\Psi_{0}$, the Kerr-Schild potential and the null tetrad vector $m$ in the twist-free case $(B=0)$ become $^{5}$ :

$$
\begin{aligned}
& \rho=-\frac{1+\cos \eta}{2 r}, \quad \sigma=-\frac{\sin \eta}{2 r}, \quad \Psi_{0}=-\frac{\sin \eta \cos \eta}{2 r^{2}} \\
& V=\frac{V_{0}}{r^{\cos \eta}}, \quad m=r^{-\frac{\cos \eta+\sin \eta+1}{2}}\left(Q_{\mathbf{1}}+i r^{\sin \eta} Q_{\mathbf{2}}\right) .
\end{aligned}
$$

where $Q_{\mathbf{1}, \mathbf{2}}=Q_{\mathbf{1}, \mathbf{2}}^{j} \frac{\partial}{\partial x^{j}}$. The functions $\eta, Q_{\mathbf{1}}^{j}, Q_{\mathbf{2}}^{j}$ and $V_{0}$ do not depend on $r$, the affine parameter of the (geodesic) integral curves of $l=D=\frac{\partial}{\partial r}$. The tetrad has been uniquely fixed by choosing

$$
\epsilon=0, \quad \pi=\alpha+\bar{\beta}, \quad m^{0}=0
$$

The fields with $\eta=0$ are algebraically special.

Our main theorem ${ }^{4}$ can be stated in the twist-free case again:

Theorem on twist-free vacuum-vacuum Kerr-Schild maps: Unless sin $0, \pm 1, \pm \frac{1}{\sqrt{2}}$, the Kerr-Schild potential is restricted by the relation $\delta V=0$.

The treatment of the metrics with $\sin \eta=0, \pm 1, \pm \frac{1}{\sqrt{2}}$ in Ref. 5 holds unchanged for curl-free fields. In what follows, we will consider the case where the shear is not restricted. The explicit $r$ dependence of $\delta V$ is known by (1). Hence we obtain:

$$
\delta V_{0}=\delta \eta=0
$$


Though the forms of the spin coefficients (1) are simple under the curl-free condition, the integration functions $V_{0}$ and $\eta$ are not constant. (Unlike in the generic case $\bar{\rho}-\rho \neq 0$, it does not follow from the commutator $[\bar{\delta}, \delta]$ that their $\Delta$ derivatives vanish.)

The field equations are the Newman-Penrose equations and the vacuum Einsteinequations for Kerr-Schild metrics ${ }^{4}$. The subsystem of equations containing $D$ derivatives of spin coefficients $\alpha, \beta, \tau, \pi$ and $\Psi_{1}$ is closed and has been referred to as the $\Psi_{1}$ system $^{3,4}$. The general solution for this system was obtained as a finite series in the complex phase factor $C=\frac{r^{\cos \eta}-i B}{r^{\cos \eta}+i B}$. For the present nontwisting case the function $B=0$, thus $C=1$. When we take the limit $B \rightarrow 0$ for the four fundamental solutions of the $\Psi_{1}$ system, two of them vanish because they are proportional to $C-1$. The fundamental solutions can be multiplied by arbitrary $r$-independent real functions. Thus we divide by $B$ and apply the l'Hospital rule, $\frac{C-1}{B} \rightarrow-\frac{2 i}{r^{\cos \eta}}$ to obtain the two missing fundamental solutions of the $\Psi_{1}$ system. The general solution is:

$$
\begin{gathered}
\pi=\frac{E_{1} \pi^{(1)}}{r \frac{\cos \eta+\sin \eta+1}{2}}+\frac{E_{2} \pi^{(2)}}{r \frac{\cos \eta-\sin \eta+1}{2}}+\frac{E_{3} \pi^{(3)}}{r^{\frac{3 \cos \eta+\sin \eta+3}{2}}}+\frac{E_{4} \pi^{(4)}}{r^{\frac{3 \cos \eta-\sin \eta+3}{2}}} \\
\tau=\frac{E_{1} \tau^{(1)}}{r \frac{\cos \eta+\sin \eta+1}{2}}+\frac{E_{2} \tau^{(2)}}{r^{\frac{\cos \eta-\sin \eta+1}{2}}}+\frac{E_{3} \tau^{(3)}}{r^{\frac{3 \cos \eta+\sin \eta+3}{2}}}+\frac{E_{4} \tau^{(4)}}{r^{\frac{3 \cos \eta-\sin \eta+3}{2}}} \\
\alpha=\frac{E_{1} \alpha^{(1)}}{r \frac{\cos \eta+\sin \eta+1}{2}}+\frac{E_{2} \alpha^{(2)}}{r^{\frac{\cos \eta-\sin \eta+1}{2}}}+\frac{E_{3} \alpha^{(3)}}{r^{\frac{3 \cos \eta+\sin \eta+3}{2}}}+\frac{E_{4} \alpha^{(4)}}{r \frac{3 \cos \eta-\sin \eta+3}{2}} \\
\Psi_{1}=\frac{E_{1} \Psi_{1}^{(1)}}{r \frac{\cos \eta+\sin \eta+3}{2}}+\frac{E_{2} \Psi_{1}^{(2)}}{r^{\frac{\cos \eta-\sin \eta+3}{2}}}+\frac{E_{3} \Psi_{1}^{(3)}}{r^{\frac{3 \cos \eta+\sin \eta+5}{2}}}+\frac{E_{4} \Psi_{1}^{(4)}}{r^{\frac{3 \cos \eta-\sin \eta+5}{2}}}
\end{gathered}
$$

where $E_{1}, \ldots E_{4}$ are arbitrary functions independent of $r$ and the coefficients $\pi^{(1)}, \ldots$, $\pi^{(4)}, \tau^{(1)}, \ldots \tau^{(4)}, \alpha^{(1)}, \ldots \alpha^{(4)}, \Psi_{1}^{(1)}, \ldots \Psi_{1}^{(4)}$ enlisted in Table 1 are functions of $\eta$ only.

Once the affine-parameter dependence of the spin coefficients $\rho, \sigma, \alpha, \beta, \pi, \tau, \Psi_{0}, \Psi_{1}$ and of the Kerr-Schild potential is known, the question naturally arises whether these solutions are compatible with the rest of the equations? In the following section, we will address this question. 


\section{THE REMAINING FIELD EQUATIONS}

First we find from the commutator $[\bar{\delta}, \delta] r=\mu-\bar{\mu}$ that the spin coefficient $\mu$ is real. Then, writing the tetrad vector $n$ in the form

$$
n=\mathcal{N} \partial / \partial r+N^{j} \partial / \partial x^{j}
$$

where $\mathcal{N}, N^{j}$ are unknown functions of all of the coordinates, some $\Delta$ derivatives can be written as:

$$
\begin{aligned}
\Delta \rho & =\frac{1+\cos \eta}{2 r^{2}} \mathcal{N}+\frac{\sin \eta}{2 r} \Delta \eta, \quad \Delta \sigma=\frac{1+\cos \eta}{2 r^{2}} \mathcal{N}+\frac{\sin \eta}{2 r} \Delta \eta \\
\Delta \Psi_{0} & =\frac{\sin \eta \cos \eta}{r^{3}} \mathcal{N}-\frac{\cos ^{2} \eta-\sin ^{2} \eta}{2 r^{2}} \Delta \eta, \quad \Delta \ln V=\Delta \ln V_{0}-\frac{\cos \eta}{r} \mathcal{N}+\sin \eta \ln r \Delta \eta .
\end{aligned}
$$

The equations (NP 4.2.1,q) of Ref. 6, the complex conjugate of (NP 4.2.p), the fifth Bianchi relation (NP 4.5) and the last Kerr-Schild equation ${ }^{3,5}$ form a closed algebraic system in the real variables $\mu, \mathcal{N}, \Delta \eta, \Delta l n V_{0}$ and the complex variables $\lambda, \gamma, \Psi_{2}$ and their complex conjugates:

$$
\begin{array}{r}
\rho \mu-\sigma \lambda-\Psi_{2}=a_{1} \\
\sigma \mu+\rho \lambda+\sigma(\gamma-3 \bar{\gamma})+\frac{1+\cos \eta}{2 r^{2}} \mathcal{N}+\frac{\sin \eta}{2 r} \Delta \eta=\bar{a}_{2} \\
\rho \mu+\sigma \lambda-\rho(\gamma+\bar{\gamma})+\Psi_{2}+\frac{\sin \eta}{2 r^{2}} \mathcal{N}-\frac{\cos \eta}{2 r} \Delta \eta=a_{3} \\
\Psi_{0} \mu-4 \Psi_{0} \gamma-3 \sigma \Psi_{2}+\frac{\sin \eta \cos \eta}{r^{3}} \mathcal{N}-\frac{\cos ^{2} \eta-\sin ^{2} \eta}{2 r^{2}} \Delta \eta=a_{4} \\
\frac{\Psi_{0}}{\sigma} \mu-2 \rho(\gamma+\bar{\gamma})+\Psi_{2}+\bar{\Psi}_{2}-\rho\left(\Delta \ln V_{0}-\frac{\cos \eta}{r} \mathcal{N}+\sin \eta \ln r \Delta \eta\right)
\end{array}
$$

where the source terms $a_{1}, \ldots a_{5}$ are given in Table 2, and their $r$ dependence is known. This system corresponds to, but does not hold as a limiting case, of Eqs. (5.2) of Ref. 5 . The unknown functions $\mathcal{N}, \Delta \eta$ and $\Delta \ln V_{0}$ did not occur in the generic treatment. Now we cannot get rid of $\mathcal{N}$ by use of the commutator $[\delta, \bar{\delta}] r$ because $(\bar{\rho}-\rho) \mathcal{N}$ vanishes, and we cannot prove property (ii) (that the $\Delta$ derivatives vanish). 
The imaginary parts of the first four equations of (7) yield:

$$
\begin{aligned}
\lambda-\bar{\lambda} & =\frac{-3 \sigma^{2}\left(a_{1}-\bar{a}_{1}\right)+\sigma\left(a_{4}-\bar{a}_{4}\right)-\Psi_{0}\left(a_{2}-\bar{a}_{2}\right)}{3 \sigma^{3}+\rho \Psi_{0}} \\
\gamma-\bar{\gamma} & =-\frac{a_{2}-\bar{a}_{2}+\rho(\lambda-\bar{\lambda})}{4 \sigma} \\
\Psi_{2}-\bar{\Psi}_{2} & =-\sigma(\lambda-\bar{\lambda})-\left(a_{1}-\bar{a}_{1}\right) \\
C_{1} & \equiv a_{1}+a_{3}-\bar{a}_{1}-\bar{a}_{3}=0
\end{aligned}
$$

The last relation is a constraint equation involving only expressions with known $r$ dependence.

With algebraic manipulations on the first four equations of (7) one obtains:

$$
\begin{aligned}
\mu & =\frac{1+\cos \eta}{\sin \eta} \lambda+f_{1} \\
r \Psi_{2} & =-\frac{\cos \eta(1+\cos \eta)}{\sin \eta} \lambda+f_{2} \\
\gamma+\bar{\gamma}+\frac{\mathcal{N}}{r} & =\frac{2(1+\cos \eta)}{\sin \eta} \lambda+f_{3} \\
\Delta \eta & =\frac{2 r\left(a_{1}+a_{2}\right)+(1+\cos \eta)\left(2 f_{1}-f_{3}\right)}{\sin \eta} \equiv r C_{2}+r^{2} C_{3}
\end{aligned}
$$

where $f_{1}, f_{2}, f_{3}$ enlisted in Table 3 were formed from $a_{1}, \ldots a_{4}$. Note that the commutator $[\Delta, D] \eta$ implies that $D \eta$ does not depend on $r$, so $\Delta \eta=0$ and $C_{2}=C_{3}=0$ are constraints.

From the equations (NP 4.2.g) the $r$ dependence of $\lambda$ can be integrated:

$$
D\left(r^{1+\cos \eta} \lambda\right)=r^{1+\cos \eta}\left(\sigma f_{1}+f_{4}\right)
$$

where the term $f_{4}$ with known $r$ dependence is given in Table 3 . After the elimination of $\lambda$ from (10) and (NP 4.2.h), a fourth constraint $C_{4}=0$ arises. The solution of all these constraints allows one to express the $Q_{\mathbf{1}, \mathbf{2}}$ derivatives of $E_{1}, E_{2}$ in terms of $E_{1}, E_{2}$, while $E_{3}, E_{4}$ is found to vanish (Table 4 ).

Finally, from $[\Delta, D] r$ one gets $\left(\gamma+\bar{\gamma}+\frac{\mathcal{N}}{r}\right)=r D\left(-\frac{\mathcal{N}}{r}\right)$, and with a second integration one finds the $r$ dependence of $\mathcal{N}$.

Using all these results in the last relation of (7), we express $\Delta \ln V_{0}$, which does not depend on $r$ as follows from the commutator $[\Delta, D] \ln V_{0}$. This condition can be fulfilled 
only if:

$$
E_{1}=E_{2}=E_{3}=E_{4}=0
$$

We recover the same result as in the general case: only the trivial solution of the $\Psi_{1}$ system is compatible with the rest of the equations. We then have

$$
\begin{aligned}
& \kappa=\epsilon=\pi=\tau=\alpha=\Psi_{1}=\mu=\lambda=\Psi_{2}=\Delta \eta=0 \\
& \gamma=-\frac{H}{2}, \quad \mathcal{N}=H r, \quad \Delta \ln V_{0}=(\cos \eta+2) H
\end{aligned}
$$

where $H$ is an integration function restricted by the conditions $Q_{\mathbf{1}} H=Q_{\mathbf{2}} H=0$ following from the commutator $[\delta, D] \ln V_{0}$.

We complete the process of determining the $r$ dependence of the spin coefficients by observing that

$$
\nu=\Psi_{3}=\Psi_{4}=0
$$

from $[\delta, D] r$ and (NP 4.2.i,j) respectively. We conclude that the base space must be of type $\mathrm{N}$.

\section{THE METRICS}

The commutators among $Q_{\mathbf{1}}, Q_{\mathbf{2}}, N=N^{j} \frac{\partial}{\partial r}$ and $D$, namely

$$
\begin{aligned}
& {[N, D]=\left[Q_{\mathbf{1}}, D\right]=\left[Q_{\mathbf{2}}, D\right]=\left[Q_{\mathbf{1}}, Q_{\mathbf{2}}\right]=0} \\
& {\left[Q_{\mathbf{1}}, N\right]=-\frac{\cos \eta+\sin \eta+1}{2} H Q_{\mathbf{1}}, \quad\left[Q_{\mathbf{2}}, N\right]=-\frac{\cos \eta-\sin \eta+1}{2} H Q_{\mathbf{2}}}
\end{aligned}
$$

allow one to fix the coordinates $(r, x, y, u)$ in the following way:

$$
\begin{aligned}
& D=\frac{\partial}{\partial r}, \quad Q_{\mathbf{1}}=\frac{\partial}{\partial x}, \quad Q_{\mathbf{2}}=\frac{\partial}{\partial y} \\
& N=-\frac{\cos \eta+\sin \eta+1}{2} H(u) \frac{\partial}{\partial x}-\frac{\cos \eta-\sin \eta+1}{2} H(u) \frac{\partial}{\partial y}+F(u) \frac{\partial}{\partial u}
\end{aligned}
$$

where $F(u)$ is an arbitrary function of $u$. The tetrad vectors in the chosen coordinates are:

$$
\begin{aligned}
& l^{a}=\delta_{1}^{a}=r^{-\frac{\cos \eta+\sin \eta+1}{2}} \delta_{2}^{a}+r^{-\frac{\cos \eta-\sin \eta+1}{2}} \delta_{3}^{a} \\
& n^{a}=u r \delta_{1}^{a}-\frac{\cos \eta+\sin \eta+1}{2} u x \delta_{2}^{a}-\frac{\cos \eta-\sin \eta+1}{2} u y \delta_{3}^{a}+F(u) \delta_{4}^{a}
\end{aligned}
$$


The Kerr-Schild potential is found by integration of $V_{0}$ from (12):

$$
V=\Lambda \frac{e^{(\cos \eta+2) \int \frac{u d u}{F(u)}}}{r^{\cos \eta}}
$$

where $\Lambda$ is an arbitrary parameter.

We are now able to write down the base space metric using the completeness relation of the tetrad (16). With (17), the Kerr-Schild pencil has the form

$$
\begin{aligned}
d s^{2}= & -\frac{r^{\cos \eta+\sin \eta+1}}{2}\left[d x+\frac{(\cos \eta+\sin \eta+1) x u}{2 F(u)} d u\right]^{2} \\
& -\frac{r^{\cos \eta-\sin \eta+1}}{2}\left[d y+\frac{(\cos \eta-\sin \eta+1) y u}{2 F(u)} d u\right]^{2} \\
& -\frac{1}{2 r u}\left[d r-\frac{2 r u}{F(u)} d u\right]^{2}+\frac{d r^{2}}{2 r u} \\
d \tilde{s}^{2}= & d s^{2}+\Lambda \frac{e^{(\cos \eta+2) \int \frac{u d u}{F(u)}}}{r^{\cos \eta}} \frac{d u^{2}}{F(u)^{2}}
\end{aligned}
$$

The curvature components are:

$$
\begin{aligned}
& \tilde{\Psi}_{0}=-\frac{\cos \eta \sin \eta}{2 r^{2}} \\
& \tilde{\Psi}_{1}=\tilde{\Psi}_{3}=0 \\
& \tilde{\Psi}_{2}=\Lambda \frac{\cos \eta(\cos \eta+1) e^{(\cos \eta+2) \int \frac{u d u}{F(u)}}}{4 r^{\cos \eta+2}} \\
& \tilde{\Psi}_{4}=-\Lambda^{2} \frac{\cos \eta \sin \eta e^{2(\cos \eta+2) \int \frac{u d u}{F(u)}}}{8 r^{2 \cos \eta+2}} .
\end{aligned}
$$

We conclude that the Kerr-Schild space-time is of type I in the Petrov classification.

We may transform these metrics to a simpler form by the coordinate transformations

$$
t=e^{\int \frac{u d u}{F(u)}}, \quad v=\frac{1}{\sqrt{2}} x t^{\frac{\cos \eta+\sin \eta+1}{2}}, \quad w=\frac{1}{\sqrt{2}} y t^{\frac{\cos \eta-\sin \eta+1}{2}}, \quad p=r t .
$$

Noting that $t$ is function only of $u$, we define a new coordinate $q$ in place of $t$ by $d q=\frac{d t}{u}$. The old coordinates $(r, x, y, u)$ can be expressed in terms of the new ones $(v, w, p, q)$ provided that the first relation of (20) is invertible. The base and Kerr-Schild metrics in these new coordinates are:

$$
\begin{aligned}
& d s^{2}=-p^{\cos \eta+s i n \eta+1} d v^{2}-p^{\cos \eta-s i n \eta+1} d w^{2}+2 d p d q \\
& d \tilde{s}^{2}=d s^{2}+\Lambda p^{-\cos \eta} d q^{2}
\end{aligned}
$$


The parent space metric has been obtained by Bilge ${ }^{7}$. There has been some controversy in the literature (cf. Kupeli $^{8}$ and Bilge ${ }^{7}$ ) whether or not this pp-wave is unique in the considered class of Kerr-Schild maps. Here we succeeded in demonstrating the uniqueness.

The vectors $\frac{\partial}{\partial v}, \frac{\partial}{\partial w}$ and $\frac{\partial}{\partial q}$ are (commuting) Killing vectors of both metrics, moreover the last of them is covariantly constant. In the shear-free limit sin $\rightarrow 0$ both metrics admit a fourth Killing vector $v \frac{\partial}{\partial w}-w \frac{\partial}{\partial v}$, thus they are plane symmetric metrics (13.9) of Ref. 9.

The metric $d \tilde{s}^{2}$ in $(21)$ can be put into a more familiar form as follows,

(a) in case $\Lambda<0$ we introduce the new coordinates $(T, X, Y, Z)$ :

$$
\begin{aligned}
& T=\frac{(-\Lambda)^{\frac{-1}{2}}}{\frac{\cos \eta}{2}+1} p^{\frac{\cos \eta}{2}+1} \\
& X=\left[(-\Lambda)^{\frac{1}{2}}\left(\frac{\cos \eta}{2}+1\right)\right]^{\frac{\cos \eta+\sin \eta+1}{2}} v \\
& Y=\left[(-\Lambda)^{\frac{1}{2}}\left(\frac{\cos \eta}{2}+1\right)\right]^{\frac{\cos \eta-\sin \eta+1}{2}} w \\
& Z=\left[(-\Lambda)^{\frac{1}{2}}\left(\frac{\cos \eta}{2}+1\right)\right]^{\frac{-\cos \eta}{2}}\left[(-\Lambda)^{\frac{1}{2}} q-(-\Lambda)^{\frac{-1}{2}} \frac{p^{1+\cos \eta}}{1+\cos \eta}\right] .
\end{aligned}
$$

The Kerr-Schild metric is the Kasner metric ${ }^{10}$ :

$$
d \tilde{s}^{2}=d T^{2}-T^{2 p_{1}} d X^{2}-T^{2 p_{2}} d Y^{2}-T^{2 p_{3}} d Z^{2}
$$

where the powers

$$
p_{1}=\frac{\cos \eta+\sin \eta+1}{\cos \eta+2}, \quad p_{2}=\frac{\cos \eta-\sin \eta+1}{\cos \eta+2}, \quad p_{3}=\frac{-\cos \eta}{\cos \eta+2}
$$

satisfy the required relations $p_{1}+p_{2}+p_{3}=1$ and $\left(p_{1}\right)^{2}+\left(p_{2}\right)^{2}+\left(p_{3}\right)^{2}=1$.

(b) in case $\Lambda>0$ the new coordinates $(T, X, Y, Z)$ are introduced in the following 
manner:

$$
\begin{aligned}
T & =\frac{(\Lambda)^{\frac{-1}{2}}}{\frac{\cos \eta}{2}+1} p^{\frac{\cos \eta}{2}+1} \\
X & =\left[(\Lambda)^{\frac{1}{2}}\left(\frac{\cos \eta}{2}+1\right)\right]^{\frac{\cos \eta+\sin \eta+1}{2}} v \\
Y & =\left[(\Lambda)^{\frac{1}{2}}\left(\frac{\cos \eta}{2}+1\right)\right]^{\frac{\cos \eta-\sin \eta+1}{2}} w \\
Z & =\left[(\Lambda)^{\frac{1}{2}}\left(\frac{\cos \eta}{2}+1\right)\right]^{\frac{-\cos \eta}{2}}\left[(\Lambda)^{\frac{1}{2}} q+(\Lambda)^{\frac{-1}{2}} \frac{p^{1+\cos \eta}}{1+\cos \eta}\right]
\end{aligned}
$$

The Kerr-Schild metric is a sign-flipped version of the Kasner metric as described by McIntosh ${ }^{11}$ :

$$
d \tilde{s}^{2}=-d T^{2}-T^{2 p_{1}} d X^{2}-T^{2 p_{2}} d Y^{2}+T^{2 p_{3}} d Z^{2}
$$

This metric arises by way of a complex coordinate transformation on the Kasner metric.

\section{CONCLUDING REMARKS}

Both solutions (23) and (26) are the special cases of the Kóta-Perjés metric (5.11) of Ref. 12 for $B=0$.

Lemma. The Kasner and sign-flipped Kasner metrics (24) and (26), respectively, are the exhaustive members of the class of vacuum Kerr-Schild metrics generated by nontwisting congruences.

Bilge $^{7}$ has considered vacuum metrics with a non-twisting geodesic congruence and subject to the conditions that $\sigma=a \rho$ and $D a=0$. He has proven that $a$ is a constant. The relation of our work with Bilge's can be established by noting that his $a=\frac{\sin \eta}{1+\cos \eta}$. Bilge's solution involves the exceptional values $\sin \eta=0, \pm 1, \pm 4 / 5$ which are different from the ones given in our Theorem. Apparently, the reason is that the Kerr-Schild property has not been imposed on the metric $\tilde{g}_{a b}$ by Bilge. 


\section{REFERENCES}

[1] Trautman, A., in Recent Developments in General Relativity, Pergamon Press ,459 (1962)

[2] Kerr, R. P. and Schild, A., Atti Del Convegno Sulla Relativit Generale: Problemi Dell' Energia e Onde Gravitazionali (Anniversary Volume, Forth Centenary of Galileo's Birth), G. Barbra, Ed. (Firenze, 1965), p. 173

[3] Gergely, Á. L., Perjés Z., Physics Letters A 181, 345 (1993)

[4] Gergely, Á. L., Perjés Z., J. Math. Phys. 35, 2438 (1994)

[5] Gergely, Á. L., Perjés Z., J. Math. Phys. 35, 2448 (1994)

[6] Newman, E. and Penrose, R., J. Math. Phys. 3, 566 (1962)

[7] Bilge, A. H., Class. Quantum Grav. 6, 823 (1989)

[8] Kupeli, A. H., Class. Quantum Grav. 5, 401 (1988)

[9] Kramer, D., et al.: Exact Solutions of Einstein Field Equations, Cambridge Univ. Press (1980)

[10] Kasner, E., Amer. J. Math. 43, 217 (1921)

[11] McIntosh, C. B. G., in Relativity Today, Z. Perjés, Ed., Nova Science Publishers, New York (1992), p. 147

[12] Kóta, J. and Perjés, Z., J. Math. Phys. 13, 1695 (1972) 


$$
\begin{aligned}
\pi^{(1)} & =-2(3 \cos \eta+4 \sin \eta)(\cos \eta-1) \cos \eta \\
\pi^{(2)} & =-2\left(4 \cos ^{2} \eta+3 \cos \eta \sin \eta-3 \cos \eta+4 \sin \eta-4\right)(\cos \eta-1) \\
\pi^{(3)} & =4\left[(\sin \eta+3) \cos \eta+(\sin \eta+1)+(\sin \eta-1) \cos ^{2} \eta-3 \cos ^{3} \eta\right] \\
\pi^{(4)} & =-4\left[(\sin \eta+1) \cos ^{2} \eta+(\sin \eta-1)+(\sin \eta-3) \cos \eta+3 \cos ^{3} \eta\right] \\
\tau^{(1)} & =2\left(\cos ^{2} \eta-2 \cos \eta \sin \eta+2 \cos \eta+2 \sin \eta-2\right)(\cos \eta-1) \\
\tau^{(2)} & =2\left(2 \cos ^{2}-\sin \eta-1\right)(\cos \eta-1) \cos \eta \\
\tau^{(3)} & =\pi^{(3)} \\
\tau^{(4)} & =-\pi^{(4)} \\
\Psi_{1}^{(2)} & =\left(7 \cos ^{3} \eta-\cos { }^{2} \eta \sin \eta+5 \cos { }^{2} \eta+10 \cos \eta \sin \eta-10 \cos \eta+8 \sin \eta-8\right)(\cos \eta-1) \\
\Psi_{1}^{(3)} & =2\left(2 \cos ^{2} \eta-4 \cos \eta \sin \eta+\cos \eta+\sin \eta-3\right)(\cos \eta+1) \cos \eta \\
\Psi_{1}^{(1)} & =-2\left(2 \cos { }^{2} \eta+4 \cos \eta \sin \eta+\cos \eta-\sin \eta-3\right)(\cos \eta+1) \cos \eta \\
\alpha^{(2)} & =2(\cos \eta+4)(\sin \eta-1)-(7 \sin \eta-11) \cos { }^{2} \eta-\cos { }^{3} \eta \\
\alpha^{(3)} & =\frac{\pi^{(3)}}{2} \\
\alpha^{(4)} & =\frac{\pi^{(4)}}{2} \\
\Psi^{2} &
\end{aligned}
$$

Table 1. The coefficients in the general solution of the $\Psi_{1}$ system

$$
\begin{aligned}
& a_{1}=\delta \alpha-\bar{\delta} \beta-\alpha \bar{\alpha}-\beta \bar{\beta}+2 \alpha \beta, \quad a_{2}=\delta \tau-\tau(\tau+\beta-\bar{\alpha}), \\
& a_{3}=\bar{\delta} \tau+\tau(\bar{\beta}-\alpha-\bar{\tau}), \quad a_{4}=\delta \Psi_{1}-\Psi_{1}(4 \tau+2 \beta) \\
& a_{5}=\delta(\bar{\tau}-\pi)+\bar{\delta}(\tau-\bar{\pi})-6 \pi \bar{\pi}+2 \pi \bar{\alpha}+2 \bar{\pi} \alpha-2 \tau \bar{\tau}-2 \alpha \tau-2 \bar{\alpha} \bar{\tau}+3 \tau \pi+3 \bar{\tau} \bar{\pi}
\end{aligned}
$$

Table 2. The source terms of eq. (7) 


$$
\begin{aligned}
& f_{1}=-\frac{2 r a_{1}}{1+c}-\frac{2 b_{1}}{s(1+c)}-\frac{2\left[s b_{2}+2 c r\left(a_{1}+a_{2}\right)\right]}{(1+c)^{2}} \\
& f_{2}=\frac{b_{1}}{s}+\frac{\left[s b_{2}+2 c r\left(a_{1}+a_{2}\right)\right]}{1+c} \\
& f_{3}=-2 r a_{1}+\frac{2 b_{1}}{s}-\frac{\left[s b_{2}+2 c r\left(a_{1}+a_{2}\right)\right]}{1+c} \\
& f_{4}=\bar{\delta} \pi+\bar{\sigma} \mu+\pi^{2}+(\alpha-\bar{\beta}) \pi
\end{aligned}
$$

where: $s=\sin \eta, \quad c=\cos \eta$,

$$
b_{1}=2 r^{2} a_{4}-2 r\left(c \bar{a}_{2}+s a_{3}\right)-4 s c(\gamma-\bar{\gamma}), \quad b_{2}=2 r \bar{a}_{2}+2 s(\gamma-\bar{\gamma})
$$

Table 3. The inhomogeneous terms in eq. $(9,10)$

$$
\begin{gathered}
\left(30 c^{5}+40 c^{4} s-12 c^{4}-16 c^{3} s+2 c^{3}-59 c^{2} s+4 c^{2}+18 c s-34 c-6 s+6\right) Q_{\mathbf{1}}\left(E_{1}\right)= \\
2 E_{1}^{2}\left(110 c^{8}-20 c^{7} s-174 c^{7}+168 c^{6} s-184 c^{6}-347 c^{5} s+546 c^{5}+127 c^{4} s\right. \\
\left.-290 c^{4}+296 c^{3} s-220 c^{3}-352 c^{2} s+340 c^{2}+152 c s-152 c-24 s+24\right)
\end{gathered}
$$

$(2 c+s-1) Q_{\mathbf{2}}\left(E_{1}\right)=-\left[Q_{\mathbf{1}}\left(E_{2}\right)(c+2 s-2)+\left(12 c s^{2}-12 c s+40 s^{4}-40 s^{3}-24 s^{2}+24 s\right) E_{1} E_{2}\right]$

$$
\begin{aligned}
& \left(7 c^{2}-24 c s-40 c+30 s+34\right) Q_{\mathbf{1}}\left(E_{2}\right)= \\
& 2 E_{1} E_{2}\left(82 c^{5}+76 c^{4} s-9 c^{4}-237 c^{3} s-289 c^{3}+136 c^{2} s+244 c^{2}\right. \\
& +36 c s-36 c-8 s+8) \\
& \left(8 c^{4}-6 c^{3} s+2 c^{3}-4 c^{2} s-5 c^{2}+6 c s+s-1\right) Q_{\mathbf{2}}\left(E_{2}\right)= \\
& 2 c^{2} E_{2}^{2}\left(10 c^{5}-20 c^{4} s-18 c^{4}+16 c^{3} s+8 c^{3}+25 c^{2} s\right. \\
& \left.\quad+10 c^{2}-25 c s-18 c+4 s+8\right)
\end{aligned}
$$

$$
E_{3}=E_{4}=0
$$

Table 4. The solution of constraints $C_{1}-C_{4}$ for $Q_{\mathbf{i}}\left(E_{k}\right), E_{3}$ and $E_{4}$ 